\title{
Adaptación y mitigación al cambio climático. Estudio de caso: Ciudadela Real de Minas, Bucaramanga, Colombia.
}

Adaptation and mitigation to climate change. Case of study: Real de Minas Citadel, Bucaramanga, Colombia.

Adaptação e mitigação às mudanças climáticas. Estudo de caso: Ciudadela Real de Minas, Bucaramanga, Colômbia.

Carlos Alberto Amaya Corredor / Unidades Tecnológicas de Santander, Colombia / camaya@correo.uts.edu.co

Carolina Hernández Contreras / Unidades Tecnológicas de Santander, Colombia / chernandez@correo.uts.edu.co

Nancy Tavera Castillo / Unidades Tecnológicas de Santander, Colombia / ntavera@correo.uts.edu.co

Natalia Alexandra Bohorquez Toledo / Unidades Tecnológicas de Santander, Colombia /nbohorquez@correo.uts.edu.co

Recibido: 17/7/2020 Aceptado: 2/11/2020 Publicado: 30/12/2020

\section{RESUMEN}

Bucaramanga es el polo de desarrollo del nororiente de Colombia; en ella el sector Ciudadela Real de Minas es una zona residencial altamente poblada y condensada. Este estudio tuvo como objetivo establecer las líneas estratégicas de acción para la adaptación y mitigación de los impactos asociados al cambio climático en dicho sector. Se tomaron como referencias las experiencias de gestión ambiental en ciudades de Europa, Suramérica y Colombia, cuya respuesta a la incidencia del cambio climático coincidiera con los elementos urbanos presentes en el enfoque de gestión territorial para contrarrestarlo en Colombia, tales como la gestión de residuos sólidos, protección y mejoramiento de las zonas verdes, mejora de la movilidad y la calidad del aire, parámetros ambientales para nuevas construcciones, y procesos de educación ambiental. Sobre esta base se diseñaron e implementaron las líneas estratégicas contextualizadas a las particularidades de la ciudadela. Se concluye que, la gestión ambiental urbana desarrolla focos de respuesta al cambio climático, por lo que su aplicación en el sector de estudio fortalece las acciones de adaptación y mitigación en ejecución, para trasformar física y socialmente el entorno y las condiciones de vida de los habitantes; las cuales pueden ser replicadas en toda la ciudad. 
Palabras clave: desarrollo sostenible, educación ambiental, gestión ambiental urbana, sostenibilidad urbana

\begin{abstract}
Bucaramanga is the development pole of northeastern Colombia. The Real de Minas Citadel sector is a highly populated and condensed residential area. The objective of this study was to establish strategically lines of action for the adaptation and mitigation of the effects of climate change in this sector. experiences of environmental management in cities of Europe, South America and Colombia were taken as a reference; which responses to the incidence of climate change make a coincidence with the urban elements present in the territorial management to nullify it in Colombia, in aspects such as solid waste management, protection and improvement of green areas, improvement of mobility and air quality, environmental parameters for new constructions. Based on this strategically lines of the citadel were developed and implemented. It was concluded that urban environmental management develops focal points of response to climate change, that it's why its application in the area of study strengthens actions in adaptation and mitigation, in execution, manage to physically and socially transform the environment and the living conditions of the inhabitants, and it can be replicated throughout the city.
\end{abstract}

Keywords: environmental education, sustainable development, urban environmental management, urban sustainability

\title{
RESUMO
}

Bucaramanga é o pólo de desenvolvimento do nordeste da Colômbia; Nele, o setor Ciudadela Real de Minas é uma área residencial densamente povoada e condensada. Este estudo teve como objetivo estabelecer as linhas estratégicas de ação para a adaptação e mitigação dos impactos associados às alterações climáticas neste setor. Foram tomadas como referência as experiências de gestão ambiental em cidades da Europa, América do Sul e Colômbia, cuja resposta à incidência das mudanças climáticas coincidiu com os elementos urbanos presentes na abordagem de gestão territorial para combatê-la na Colômbia, como a gestão de resíduos sólidos. , proteção e melhoria de áreas verdes, melhoria da mobilidade e da qualidade do ar, parâmetros ambientais para novas construções e processos de educação ambiental. Nesta base, as linhas estratégicas contextualizadas às particularidades da cidadela foram desenhadas e implementadas. Conclui-se que a gestão ambiental urbana desenvolve fontes de resposta às mudanças climáticas, portanto sua aplicação no setor de estudo fortalece as ações de adaptação e mitigação em execução, para transformar física e socialmente o meio ambiente e as condições de vida dos população; que pode ser replicado em toda a cidade.

Palavras chave: desenvolvimento sustentável, educação ambiental, gestão ambiental urbana, sustentabilidade urbana 


\section{INTRODUCCIÓN}

Según la Convención Marco de las Naciones Unidas sobre el Cambio Climático (CMNUCC), el cambio climático se relaciona con las consecuencias de la actividad humana que alteran la atmósfera mundial y que generan variabilidad del clima entre periodos de tiempo similares (Organización de Naciones Unidas [ONU], 1992). Evidencia de ello es la mayor frecuencia, intensidad y magnitud de eventos climáticos o cambios graduales en las temperaturas y las precipitaciones, que transforman el clima de una región.

La ciudad de Bucaramanga, en especial su sector Ciudadela Real de Minas, es una de las fuentes de emisión de gases de efecto invernadero registradas en el país, debido a su alta concentración de construcciones, congestión vehicular y baja dispersión del aire (que retiene moléculas contaminantes); estos contribuyen al calentamiento en toda el área metropolitana, afectan al ecosistema e inciden en la calidad de vida de los habitantes y los sectores económicos en lazona (Instituto de Hidrología, Meteorología y Estudios Ambientales [IDEAM], 2016). Desde el Plan Integral de Gestión del Cambio Climático Territorial del Santander (Ministerio de Ambiente y Desarrollo Sostenible, 2016), se ha planteado la necesidad de generar el conocimiento y las herramientas para incorporar este fenómeno a la gestión del desarrollo de territorios sostenibles, adaptados y resilientes.

Consecuentemente, la presente investigación tuvo como objetivo establecer las líneas estratégicas de acción para la mitigación y adaptación de los impactos asociados al cambio climático en la Ciudadela Real de Minas como sector significativo dentro de dicha ciudad. El estudio se realizó identificando las experiencias sobre la gestión ambiental en aspectos asociados al cambio climático, en zonas urbanas; y la consulta de fuentes secundarias de información, para identificar las variables que afectan la calidad de vida urbana y sobre las cuales se requería intervenir en la zona de estudio.

En este sentido, sirvieron como antecedentes internacionales y referencias de la investigación, los trabajos realizados por: Barton (2009), Morán (2010), Ayuntamiento de Murcia (2012), Sánchez (2013), Acitores y García (2015), Delgado et al. (2015), de Rauglaudre (2015), Robrecht (2019), Margulis (2016), Nahuelhual et al. (2016), Gobierno de la Ciudad de Buenos Aires (2017), Agencia Francesa de Desarrollo (AFD, 2018), Bravo (2018) y Sengupta (2019), entre otros.

Asimismo, como antecedentes y referentes nacionales, se ubican las investigaciones Ilevadas a cabo por: Alcaldía de Bucaramanga (2015), IDEAM (2016), Pérez (2016), Amaya et al. (2017), Veeduría Distrital (2019), Universidad Industrial de Santander (2018), Cariñanos et al. (2018) y Amaya et al. (2019). Con este estudio se presenta un enfoque estratégico pertinente a las características físicas y sociales de la ciudad de Bucaramanga, en el cual se reafirma que la atención al cambio climático debe ser una prioridad en la ejecución de sus políticas públicas, por lo que es importante fortalecer $y$ enriquecer las acciones que se vienen desarrollando para mejorar las condiciones de vida urbana, como una alternativa de solución local a una de las problemáticas globales más relevantes en la actualidad. 


\section{MATERIALES Y MÉTODOS}

El proyecto de investigación presentado como caso de estudio es de tipo cualitativo, descriptivo, explicativo (Hernández et al., 2014) y de carácter no experimental. Se buscó describir las condiciones de la infraestructura urbana de la Ciudadela Real de Minas para proponer las estrategias de adaptación y mitigación ante el cambio climático.

Dicho territorio es identificado como la Comuna 7 en la división política administrativa de Bucaramanga, y se ubica en el sector centro oriental de ella, donde radicó hasta 1976 el aeropuerto Gómez Niño. La zona fue desarrollada a partir de la estructura residencial denominada Plaza Mayor, ícono de la arquitectura colombiana en la década de los ochenta, la cual sirvió como eje para la expansión urbana sobre las 80 ha que aproximadamente abarca ese terreno.

Según el Censo poblacional y de vivienda de Colombia (Departamento Nacional de Estadística, 2018), en estelugar seencuentran cerca de 25900 habitantes y 5590 unidades habitacionales. Desde el año 2000, la Alcaldía proyectó y consolidó un espacio enfocado como ciudadela estudiantil, donde se encuentran siete colegios públicos, con más de 13000 estudiantes hasta el 2019; y tres universidades, con cerca de 17000 personas circulantes. Estos establecimientos educativos aportan al sector una población flotante cercana a 30000 personas, no todas habitantes-residentes, pero que diariamente conviven en él por cuestiones laborales o estudiantiles (Amaya et al., 2017). La infraestructura urbana está constituida por dos parques zonales públicos: Los Sueños y Las Cigarras; y los ejes viales principales: calles 61 y calle 56, avenida Juan Pablo ॥ y la avenida Los Samanes, a las cuales se conectan las vias secundarias y por la que transita todo el tráfico vehicular en el área.

El estudio revisó las doce variables de análisis de ciudades para interpretar las condiciones de mitigación y adaptación al cambio climático, establecidas por la Agencia Europea de Medio Ambiente (AEMA, 2018) teniendo en cuenta los criterios de autoridades mundiales o regionales como la CMNUCC (ONU, 1992) y los resultados de la Conferencia de las Naciones Unidas sobre la Vivienda y el Desarrollo Urbano Sostenible, Hábitat III (ONU, 2017); así como de la descripción de Oltra y Marín (2013) y Margulis (2016) sobre las respuestas urbanas ante el cambio climático.

Se estimaron las investigaciones realizadas en ciudades de Europa, Suramérica y Colombia, en virtud de reducir las doce variables propuestas por la AEMA (2018), a aquellas que tuvieran un mayor índice de coincidencia en dichas investigaciones; ello con el fin de establecer los indicadores concretos que se asumirían en el presente estudio y, consecuentemente, establecer su estructura de análisis.

En este sentido, de acuerdo con las intervenciones y objetivos clasificados por Oltra y Marín (2013); de la clasificación de elementos urbanos frente al cambio climático como condicionantes de la consolidación de una ciudad, dada por Nahuelhual et al. (2016); y de los indicadores propuestos por la AEMA (2018), se lograron definir las cinco variables que constituirían los ejes centrales del presente estudio, los cuales están en concordancia con el 
enfoque de gestión territorial al cambio climático en Colombia, establecidos por el IDEAM (2016) y el Ministerio de Ambiente y Desarrollo Sostenible (2016), mediante dos elementos: Ias condiciones de vulnerabilidad del territorio -que permitió establecer los aspectos de ciudad a intervenir en la Ciudadela Real de Minas; y las estrategias y acciones de implementación de respuestas ante el cambio climático, -que permitió enfocar, desde esta propuesta, las estrategias de respuesta a la incidencia de este-.

Para caracterizar las variables seleccionadas se recorrió la zona de estudio y se tomó la información de fuentes secundarias, tales como la Alcaldía de Bucaramanga (2015), la Corporación para la Defensa de la Meseta de Bucaramanga (2014) como autoridad ambiental territorial y el Área Metropolitana de Bucaramanga (AMB, 2019) como entidad de gestión territorial. Aquí se estableció la relación que podía darse entre la infraestructura de la zona, las cinco variables definidas y sus posibilidades de adecuarse para responder a la incidencia del cambio climático.

Con los argumentos emitidos por Sánchez (2013) y algunos lineamientos de la Nueva Agenda Urbana, generada en Hábitat III (ONU, 2017), se propusieron intervenciones en periodos de tiempo que, según el Grupo Intergubernamental de Expertos sobre el Cambio Climático (IPCC, 2015), deben apuntar a mejorar la vulnerabilidad del mundo ante este fenómeno; se mencionan como resultados a corto plazo, la adaptación de alcance local, y como resultados a largo plazo, la mitigación a nivel global.

\section{RESULTADOS}

Como fue presentado en la Conferencia de las Naciones Unidas sobre Cambio Climático (COP 25) (ONU, 2019), las ciudades son las áreas que más aportan al incremento de las consecuencias del cambio climático, por aspectos como el aumento de la densidad poblacional, las emisiones por fuentes móviles y fijas, y el uso indiscriminado de los recursos. Sobre esta base se tomaron los parámetros de análisis de la AEMA (2018) y de Sánchez (2013), para identificar en las ciudades acciones contra el cambio climático; los cuales se enfocaron en doce variables: agricultura, biodiversidad, edificios, áreas costeras, reducción de riesgos de desastre, energía, silvicultura, gobernanza y soporte financiero, salud, transporte, urbanismo y gestión del agua.

Cabe puntualizar que en el análisis de las mismas, las acciones evidenciadas por ciudades no solo se limitaron a la denominación específica de ellas, según la AEMA (2018), sino que también se contemplaron las acciones que pueden incluirse dentro de sus componentes. Tomando en cuenta la trascendencia que para la ONU (2017) tiene la Nueva Agenda Urbana, Hábitat III, es importante destacar que estas variables guardan estrecha relación con el principio de garantizar la sostenibilidad del ambiente, en el cual se enmarcan aspectos como: los ecosistemas y la diversidad biológica, los estilos de vida saludable, el consumo y la producción sostenible, la reducción de riesgos de desastre, las prácticas de adaptación al cambio climático y la mitigación de 
sus efectos en la consolidación de las ciudades (ONU, 2017). Los autores de esta investigación aclaran que no se incluyen en ella datos referentes a áreas costeras, al ubicarse geográficamente Bucaramanga en la ramificación nororiental del macizo montañoso del país.

En Europa, la mayoría de las ciudades tienen planes de adaptación muy avanzados Robrecht (2019). Estas poseen un número considerable de habitantes, que condiciona el hecho de que, en las políticas de gestión urbana, se incluyan la capacidad que deben tener para hacer frente a este fenómeno global. La Comisión Europea describe experiencias exitosas de urbes frente al cambio climático en su línea de trabajo ciudades y desarrollo urbano, tales como Växjö, Suecia (de Rauglaudre, 2015); Copenhague, Dinamarca (Comisión Europea, 2010; Sengupta, 2019); Ámsterdam, Holanda (Acitores y García, 2015; Bastein et al., 2018); Róterdam, Holanda (Ecointeligencia, 2012); Bolonia, Italia (Morán, 2010); Valencia, España (Muévete en Verde, 2018); y Murcia,
España (Ayuntamiento de Murcia, 2012).

Con esta información se realizó una matriz para confrontar el objetivo e impacto de las acciones realizadas en dichas ciudades, con las variables propuestas por la AEMA (2018), de acuerdo a su coincidencia entre las ciudades y el enfoque de sus acciones ante el cambio climático.

En la tabla 1 se describe que, de las once variables analizadas, solo siete ciudades desarrollan acciones en dos; cinco ciudades desarrollan acciones en cuatro; solo tres ciudades coinciden en la ejecución de las acciones, en dos; y de una variable solo se reconocen acciones en una ciudad. En una variable ninguna ciudad decidió enfocar sus acciones. Estas coincidencias permitieron identificar a: la biodiversidad, la silvicultura, la gobernanza, el transporte, el urbanismo y la salud, como las variables de mayor relevancia para estas urbes europeas, al proponerse en aquellas el mayor número de acciones de respuesta al cambio climático.

Tabla 1. Relación de actividades de ciudades europeas con sectores de la AEMA.

\begin{tabular}{|c|c|c|c|c|c|c|c|c|c|c|c|}
\hline Ciudad & 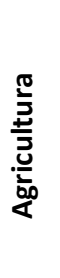 & $\begin{array}{l}\frac{0}{\pi} \\
\frac{\pi}{0} \\
\frac{0}{2} \\
\frac{2}{0} \\
\frac{0}{0} \\
\frac{0}{0}\end{array}$ & 魚 & 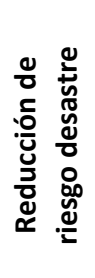 & $\frac{\frac{\pi}{00}}{\frac{0}{\omega}}$ & 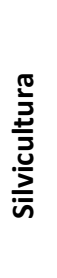 & 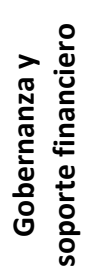 & $\frac{\text { 음 }}{\sqrt{n}}$ & 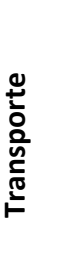 & 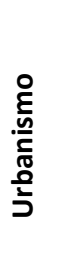 & 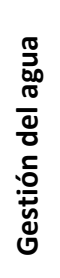 \\
\hline Växjö & & $x$ & $x$ & $x$ & $x$ & $X$ & $\mathrm{X}$ & & $x$ & & \\
\hline Copenhague & & $x$ & & $x$ & $x$ & $X$ & $x$ & $x$ & $x$ & $x$ & \\
\hline Ámsterdam & & $x$ & $x$ & & & $x$ & $x$ & $x$ & $x$ & $x$ & \\
\hline Róterdam & & $x$ & $x$ & & & $x$ & $x$ & & $x$ & & \\
\hline Bolonia & & $x$ & $x$ & & & $x$ & $x$ & $x$ & & $\mathrm{X}$ & \\
\hline Valencia & & $x$ & & & & $x$ & $x$ & $x$ & $x$ & $x$ & \\
\hline Murcia & & $x$ & & $x$ & $x$ & $x$ & & $x$ & $x$ & $x$ & $\mathrm{x}$ \\
\hline $\begin{array}{l}\text { Ciudades } \\
\text { coincidentes } \\
\text { en variable }\end{array}$ & 0 & 7 & 4 & 3 & 3 & 7 & 6 & 5 & 6 & 6 & 1 \\
\hline
\end{tabular}

Fuente: Elaboración propia, con base en las variables de la AEMA(2018). 
A nivel de Suramérica (Delgado et al., 2015; Margulis, 2016), las principales experiencias a referenciar se tomaron de ciudades como Curitiba (AFD, 2018) y Brasilia (Bravo, 2018) en Brasil; Quito (Organización de las Naciones Unidas para la Alimentación y la Agricultura, 2016) en Ecuador; Santiago de Chile (Barton, 2009) en Chile; y Buenos Aires (Gobierno de la Ciudad de Buenos Aires, 2017) en Argentina. Al contrastar las acciones de las ciudades con las variables de la AEMA (2018) propuestas, se logró clasificarlas de acuerdo con la coincidencia entre ciudades y del enfoque de sus acciones ante el cambio climático.
Hay que destacar que de las once variables: las cinco ciudades desarrollan acciones en dos; tres de las ciudades desarrollan acciones en cuatro; solo dos ciudades coinciden en ejecución de acciones, en dos; sobre dos variables solo se recopiló acciones en una ciudad; y en una variable ninguna ciudad evidenció enfocar acciones. Estas coincidencias permitieron identificar como las variables más destacadas entre las ciudades de Suramérica a: la silvicultura, la salud, la gobernanza, el urbanismo, el trasporte y la construcción de edificios; en las que se proponen acciones concretas de respuesta al cambio climático (tabla 2).

Tabla 2. Relación de actividades de ciudades suramericanas con sectores de la AEMA.

\begin{tabular}{|c|c|c|c|c|c|c|c|c|c|c|}
\hline $\begin{array}{c}\text { Variable } \\
\text { AEMA }\end{array}$ & 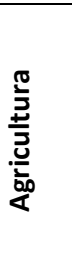 & 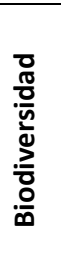 & $\frac{\stackrel{n}{u}}{: \underline{\underline{t}}}$ & 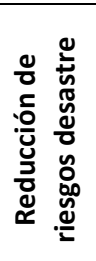 & $\frac{\frac{\pi}{00}}{\frac{0}{20}}$ & $\begin{array}{l}\frac{\pi}{3} \\
\frac{\pi}{3} \\
\frac{\pi}{3} \\
\frac{\pi}{n}\end{array}$ & 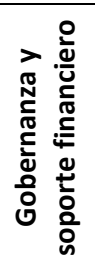 & $\frac{\frac{0}{\pi}}{\sqrt[N]{n}}$ & 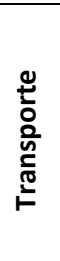 & 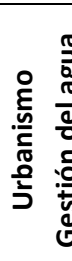 \\
\hline Curitiba & $x$ & $\mathrm{x}$ & $x$ & $x$ & & $x$ & $x$ & $x$ & & \\
\hline Brasilia & & $\mathrm{x}$ & & & & $\mathrm{x}$ & $\mathrm{x}$ & $\mathrm{x}$ & $\mathrm{x}$ & $\mathrm{x}$ \\
\hline Quito & $x$ & & $x$ & & & $x$ & $x$ & $x$ & & $x$ \\
\hline Santiago de Chile & & & & & & $\mathrm{x}$ & $\mathrm{x}$ & $\mathrm{x}$ & $\mathrm{x}$ & $\mathrm{x}$ \\
\hline Buenos Aires & & & $x$ & & $x$ & $x$ & & $x$ & $x$ & $x$ \\
\hline $\begin{array}{l}\text { Ciudades } \\
\text { coincidentes } \\
\text { en variable }\end{array}$ & 2 & 2 & 3 & 1 & 1 & 5 & 4 & 5 & 3 & 4 \\
\hline
\end{tabular}

Fuente: Elaboración propia, con base en las variables de la AEMA(2018).

Por su parte, en el Plan nacional de adaptación al cambio climático, Colombia (Departamento Nacional de Planeación, 2012) propone las bases parasuadaptación y mitigación ante este fenómeno. A partir de dicho documento rector y de los Objetivos de Desarrollo Sostenible (Universidad Industrial de Santander, 2018) que impulsan la Agenda 2030 (ONU, 2015), el Ministerio de Ambiente y
Desarrollo Sostenible (2016) y la Alcaldía de Bucaramanga han identificado la línea base de su trabajo para la formulación y puesta en marcha de acciones locales, que aporten a la gestión del calentamiento global.

Se tomaron como ciudades referencias: Bogotá, capital del país y referente urbano nacional (Veeduría Distrital, 2019); Medellín, por su trasformación sostenible 
(Pérez, 2016; Área Metropolitana del Valle de Aburra, 2019); e Ibagué, por su constitución física y social, similar a Bucaramanga (Corporación Autónoma Regional del Tolima, 2018). Al contrastar las acciones de estas ciudades con las variables de la AEMA (2018) propuestas, se logró clasificarlas de acuerdo con la coincidencia entre las ciudades y el enfoque de sus acciones ante el cambio climático.

En la tabla 3 se evidencia que, de las once variables: las tres ciudades desarrollan acciones en cinco; solo dos ciudades coinciden en ejecución de acciones, en dos; sobre dos variables solo se recopiló acciones en una ciudad; $y$ en dos variables ninguna ciudad planteó enfocar acciones. Estas coincidencias permitieron identificar como las variables más destacadas, entre las ciudades de Colombia a: la biodiversidad, la silvicultura, la salud, la gobernanza y el trasporte; en las que se proponen acciones de respuesta al cambio climático.

Tabla 3. Relación de actividades de ciudades colombianas con sectores de la AEMA.

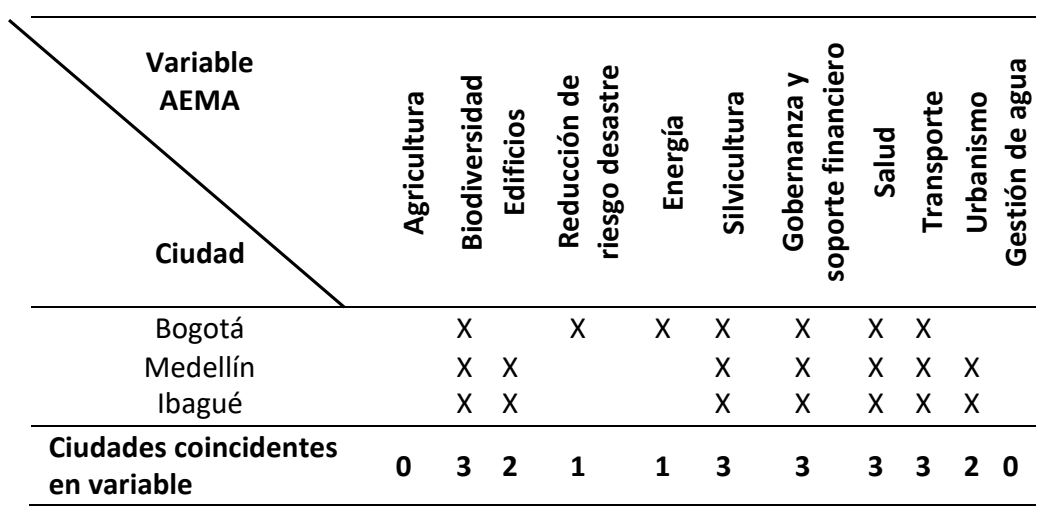

Fuente: Elaboración propia, con base en las variables de la AEMA(2018).

La revisión de la información integrada permitió comprobar que, en las 15 ciudades documentadas, los sectores urbanos que reúnen más acciones para responder al cambio climático son: la biodiversidad, los edificios, la silvicultura, la gobernanza, la salud, el transporte y el urbanismo; mientras que la agricultura, la gestión del agua, la gestión del riesgo de desastres y las energías, no poseen acciones significativas. Es importante destacar que se comprobó la relación de las variables establecidas por la AEMA (2018), identificadas como comunes, con los aspectos recogidos en la Nueva
Agenda Urbana, Hábitat III (ONU, 2017); debido a que este último, en sus compromisos de aplicación efectiva para la consolidación de ciudades sostenibles, promueve desde el desarrollo urbano resiliente y ambientalmente sostenible, la planificación y gestión del desarrollo espacial urbano en el diseño de servicios e infraestructura de construcciones y edificios, con soluciones basadas en la naturaleza; infraestructuras sostenibles de movilidad y transporte; así como la mejora de la salud a través de la gestión del agua, la reducción de residuos y el fortalecimiento de los gobiernos. 
Las variables resultantes fueron contrastadas con los elementos para la consolidación de una ciudad frente al cambio climático, establecidos por Nahuelhual et al. (2016); y relacionadas con los lineamientos presentes en el enfoque de gestión territorial al cambio climático en Colombia (IDEAM, 2016; Ministerio de Ambiente y Desarrollo
Sostenible, 2016), desde la descripción de las vulnerabilidades del territorio y en función de establecer los aspectos de la ciudad a intervenir en el sector de la Ciudadela Real de Minas; para desde las estrategias y acciones de implementación, enfocar las líneas estratégicas de respuesta ante la incidencia del cambio climático en ella.

Tabla 4. Relación metodológica de enfoque de acciones por componentes en la zona de estudio.

\begin{tabular}{|c|c|c|c|}
\hline AEMA & $\begin{array}{l}\text { Nahuelhual et al. } \\
\qquad(2016)\end{array}$ & $\begin{array}{l}\text { IDEAM- Ministerio de } \\
\text { Ambiente y Desarrollo } \\
\text { Sostenible }\end{array}$ & $\begin{array}{l}\text { Aspectos de ciudad en } \\
\text { la Ciudadela Real } \\
\text { de Minas }\end{array}$ \\
\hline $\begin{array}{l}\text { Biodiversidad } \\
\text { Silvicultura }\end{array}$ & $\begin{array}{l}\text { Costumbre y calidad } \\
\text { de vida del ciudadano }\end{array}$ & $\begin{array}{c}\text { Espacios verdes, ecosistemas } \\
\text { urbanos y entornos de vida }\end{array}$ & $\begin{array}{c}\text { Pérdida de espacios } \\
\text { verdes y vegetación } \\
\text { urbana }\end{array}$ \\
\hline $\begin{array}{l}\text { Edificios } \\
\text { Urbanismo }\end{array}$ & $\begin{array}{l}\text { Consolidación de } \\
\text { ciudad }\end{array}$ & $\begin{array}{c}\text { Construcciones ecológicas e } \\
\text { infraestructura urbana y } \\
\text { entornos de vida }\end{array}$ & $\begin{array}{c}\text { Aumento y } \\
\text { densificación de las } \\
\text { construcciones en } \\
\text { propiedad horizontal }\end{array}$ \\
\hline $\begin{array}{l}\text { Gobernanza y } \\
\text { soporte } \\
\text { financiero }\end{array}$ & $\begin{array}{l}\text { Comercio, bienes y } \\
\text { servicios }\end{array}$ & $\begin{array}{l}\text { Educación ciudadana, } \\
\text { gestión de residuos sólidos, } \\
\text { agua y servicios públicos }\end{array}$ & $\begin{array}{c}\text { Inadecuados procesos } \\
\text { para la gestión de residuos } \\
\text { sólidos }\end{array}$ \\
\hline \multicolumn{4}{|l|}{ Salud } \\
\hline Trasporte & Movilidad & $\begin{array}{c}\text { Sistemas de Transporte y } \\
\text { calidad del aire }\end{array}$ & $\begin{array}{l}\text { Niveles de calidad del } \\
\text { aire }\end{array}$ \\
\hline
\end{tabular}

Fuente: Elaboración propia

Identificados los aspectos de la ciudad que se debían intervenir en el área de estudio, con el enfoque de Oltra y Marín (2013) y bajo los argumentos de Sánchez (2013), se describieron los principales aspectos de la zona que podían estar siendo afectados por el cambio climático:

\section{Pérdida de vegetación}

En el período 2000-2015, en la Ciudadela se trasformaron en construcciones cerca de 20 ha de espacios verdes y coberturas naturales; ya en el 2019 solo se disponía de 3 ha de su superficie para áreas verdes o coberturas naturales. Hoy ha copado sus espacios disponibles y ha reducido su arbolado urbano a limitadas zonas verdes en trazados viales y al interior de los conjuntos residenciales (Hernández et al., 2019).

\section{Aumento de la propiedad horizontal}

Según el Plan de desarrollo municipal de la ciudad, entre 2012 y 2018 en la Ciudadela aumentaron las construcciones de propiedad horizontal en un $36 \%$, así como su población, cercana a 6000 habitantes; 
lo primero ocasionó la alteración de los flujos de viento superficial, que estanca el microclima en esta zona (Alcaldía de Bucaramanga, 2015).

\section{Inadecuados procesos para la gestión de residuos sólidos}

La producción per cápita de residuos es de $0.91 \mathrm{~kg} /$ habitante/día en el sector, lo cual está por debajo del promedio municipal (0.971), pero muy por encima del promedio normativo nacional (0.76). El Plan de gestión de residuos sólidos registra que en el 2015 la Ciudadela generaba cerca de $17 \mathrm{t}$ de residuos diarias y ya en el 2019 se acercaba a las 22 t (Alcaldía de Bucaramanga, 2015).

\section{Contaminación del aire}

Este impacto se atribuye directamente al alto tráfico vehicular. Según el estudio del índice de Calidad del Aire en Bucaramanga (AMB , 2019), el sector mostró altos niveles de contaminación por material particulado PM10. Según el Plan Maestro de Movilidad (2011-2030), realizado por el AMB (2011), el $52 \%$ de la población de esa ciudad se moviliza en sistemas de trasporte público, mientras que en automóvil particular cerca del $18 \%$; el $2 \%$ lo hace a través de bicicletas o caminatas. Estos valores expresan un alto número de habitantes que hace uso del trasporte automotor, aunque crece el porcentaje de movilidad autónoma de la población.

En los cuatro aspectos anteriores se describen las características del sector estudiado, acorde a las áreas en las que deben enfocarse las estrategias de respuesta urbana ante la incidencia del cambio climático. Además, se consideró que en la Ciudadela debía trabajarse la educación ambiental como herramienta que facilita la trasformación social a favor del ambiente y que, consecuentemente, permite fortalecer el enfrentamiento a las consecuencias de aquel.

En tal sentido, según Amaya et al. (2019), el $47 \%$ de los habitantes del sector no se han informado sobre el cambio climático; el $52 \%$ manifiesta identificar el arbolado urbano como un espacio para animales y aves; y el $65 \%$ reconoce que tiene responsabilidad sobre el ambiente de la ciudad. Porcentajes que muestran una gran debilidad en el conocimiento ambiental de los ciudadanos de la Ciudadela.

\section{DISCUSIÓN}

A través de mecanismos de aplicación efectiva entre los que se destacan la gobernanza y el respeto cultural, la protección a la vivienda y la movilidad sostenible; la Nueva Agenda Urbana, Hábitat III (ONU, 2017) promueve los códigos y las normas de regulación de construcciones, la gestión sostenible de los desechos y el fortalecimiento de la biodiversidad y los espacios verdes urbanos, como respuesta de las ciudades ante el cambio climático. Teniendo en cuenta la relación de esos aspectos con las características de las ciudades analizadas, y de los parámetros planteados por el IPCC (2015), se ha construido un enfoque que fortalece la respuesta a las problemáticas identificadas en la Ciudadela Real de Minas y la formulación de las líneas estratégicas, con sus acciones para la adaptación y mitigación ante el cambio climático en este sector y la ciudad en general. 


\section{Estrategia de Ciudadela Verde}

Esta estrategia responde a las variables de biodiversidad y silvicultura analizadas; y se fundamenta sobre la importancia que les confieren a ellas las ciudades que formaron parte del estudio, las cuales se enfocaron en recomponer sus coberturas verdes e integrarlas sosteniblemente al entorno urbano. En este sentido, la propuesta diseñada para la Ciudadela Real de Minas incluyó como:

- Acciones de adaptación (en ejecución durante 2 años): La protección y restauración de los parques locales y ejes viales del sector para recomponer las coberturas forestales urbanas. Entre 2016 y 2019 el AMB ha invertido cerca de cincuenta mil millones de pesos en el fortalecimiento de la estructura ecológica principal de la ciudad; de los que se ha proyectado sobre la calle 56 el $30 \%$ de la inversión, al constituir esta la arteria vial principal de la zona.

- Acciones de mitigación (en ejecución durante 10 años): La intensificación de la siembra de árboles en el sector, que requiere grandes cantidades. Las inversiones realizadas por el AMB desde 2016 deben mantenerse hasta el año 2026 (mínimo), para que la cobertura arbórea muestre servicios ambientales de regulación favorables a la Ciudadela.

\section{Estrategias de regulación de construcciones sostenibles}

Esta estrategia se enmarca en las variables de urbanismo y edificios; y se sustenta sobre la tendencia de las ciudades estudiadas a prever las posibles condiciones climáticas y los requerimientos ecológicos en la proyección de las construcciones, como respuesta al cambio climático. Al respecto, la propuesta diseñada para la Ciudadela incluyó como:

- Acciones de adaptación (en ejecución durante 4 años): La intervención en las construcciones de la ciudad con el fin de mejorar sus jardines frontales, la silvicultura urbana y la promoción de muros y techos verdes, con el fin de recuperar la cobertura verde sobre las losas de cemento.

- Acciones de mitigación (en ejecución durante 10 años): El balance entre las nuevas construcciones y los espacios verdes. El plan de ordenamiento territorial de la ciudad establece como obligatorio que las nuevas construcciones entreguen secciones para espacios públicos, en una relación de $8 \mathrm{~m}^{2}$ por unidad habitacional construida; la mejora de este indicador debe respetar el estándar de $9 \mathrm{~m}^{2}$ por habitante, establecido por la Organización Mundial de la Salud.

\section{Estrategia de urbanizaciones más limpias}

Esta estrategia aborda las variables de saludy gobernanza; y persigue reorientar la gestión final de los residuos sólidos, a cargo de los gobiernos municipales, con el fin de reducir la influencia de ellos en el cambio climático. Sobre este tema, la propuesta diseñada para la Ciudadela incluyó como:

- Acciones de adaptación (en ejecución durante 2 años): La implementación masiva de la regla de las tres «R»: reducir, reutilizar y reciclar; para disminuir la generación per cápita de desechos sólidos urbanos a valores cercanos al promedio nacional (0.76 kg/habitante/día). Desde 
el 2017 se construyó el punto ecológico de la Ciudadela como centro de acopio selectivo de residuos, en función de disminuir en un $15 \%$ la cantidad anual de residuos entregados para trasporte y disposición final.

- Acciones de mitigación (en ejecución durante 4 años): El perfeccionamiento del proceso de selección de residuos en la fuente y la disminución de la generación en el sector. Para el 2022, el punto ecológico de la Ciudadela debe acopiar cerca del $50 \%$ de los residuos reutilizables, y disminuir la cantidad de residuos entregados a trasporte $y$ disposición en un $30 \%$.

\section{Estrategia de movilidad en la Ciudadela Real de Minas}

Esta estrategia se proyecta sobre las variables de trasporte y salud; y proyecta lograr la eficiencia de los sistemas de movilidad como componente clave de respuesta al cambio climático, al ser el trasporte el principal generador urbano de emisiones atmosféricas contaminantes. En este sentido, la propuesta diseñada para la Ciudadela incluyó como:

- Acciones de adaptación (en ejecución durante 2 años): El aumento de la cantidad de pasajeros movilizados por el sistema de trasporte masivo de la ciudad (Metrolínea) para el 2021, con el fin de minimizar la movilidad urbana automotriz del $70 \%$ a menos del $60 \%$; y el incremento de los desplazamientos a pie y en bicicleta del $22 \%$ al $30 \%$, como modalidades de movilidad poblacional.

- Acciones de mitigación (en ejecución durante 5 años): El fortalecimiento del sistema de trasporte masivo de la ciudad
(Metrolínea) y la consolidación de su red de ciclo-rutas la cual, en la zona de estudio, proyecta tener más de $5.5 \mathrm{~km}$ disponibles para la movilidad segura. Esto deberá mejorar los niveles de calidad del aire, monitoreados permanentemente por la autoridad ambiental de la ciudad.

\section{Estrategia de educación ambiental y cambio climático}

Esta es una estrategia trasversal, pues como todo proceso de respuesta al cambio climático requiere de la inclusión del ciudadano como protagonista de la construcción de su entorno, la educación ambiental permite el fortalecimiento del tejido social y el mejoramiento de la calidad de vida de los habitantes, en armonía con el ambiente. Al respecto, la propuesta diseñada para la Ciudadela incluyó como:

- Acciones de adaptación (en ejecución durante 2 años): El fortalecimiento de los proyectos ambientales escolares en los colegios del sector, y la creación de cátedras de educación ambiental y cambio climático. En estas acciones se involucran a los siete colegios de la zona de estudio, cuya matrícula se cerca a los 13000 estudiantes, quienes pueden actuar como promotores ambientales en la solución de las problemáticas de la zona.

- Acciones de mitiqación (en ejecución durante 5 años): La masificación de los procesos de educación ambiental ciudadana en el sector, llegando por lo menos al $60 \%$ de la población residente de la zona (cerca de 15000 personas), apoyados por las entidades educativas de su entorno, con el fin de reducir los agentes contaminantes y reforzar 
la siembra de árboles como iniciativa ciudadana.

\section{CONCLUSIONES Y RECOMENDACIONES}

El estudio estableció cinco líneas estratégicas de acción como respuesta a las incidencias del cambio climático en el sector Ciudadela Real de Minas, enmarcadas en la visión de la gestión ambiental urbana, presente en las experiencias que le sirvieron de referencias internacionales y nacionales a la investigación. La inclusión particular de la educación ambiental como eje transversal de dichas líneas estratégicas, refuerza la importancia de establecer acciones que impacten directamente en el ciudadano y fortalezcan la vida urbana sosteniblemente, sobre todo en Colombia.

Al desarrollar la gestión ambiental urbana, focos de respuesta al cambio climático, su aplicación en el sector de estudio fortaleció las acciones de adaptación y mitigación, en ejecución desde la Alcaldía de Bucaramanga, para trasformar física y socialmente el entorno y las condiciones de vida de los habitantes; las cuales pueden ser replicadas en toda la ciudad.

\section{REFERENCIAS BIBLIOGRÁFICAS}

Acitores, A.S., y García, L. (2015, diciembre). Desarrollo sostenible en Smart Cities: Ámsterdam como ejemplo. Antonio Serrano Acitores. https://bit.ly/3nwklw1

Agencia Europea de Medio Ambiente. (2018). 10 Case Stduies How Europe is adapting to climate change. https://bit.ly/3azY25v

Agencia Francesa de Desarrollo. (2018, 16 de octubre). 5 ciudades pioneras en acción climática. https://bit.ly/38Lh1cl

Alcaldía de Bucaramanga. (2015). Plan de Gestión Integral de Residuos Sólidos PGIR. Formulación y actualización. https://bit.ly/38QL6Hs

Amaya, C.A., Abreo, A.L., Rey, D.C., y Ríos, D.C. (2019). Propuesta para la reducción de aportes de gases efecto invernadero, asociados a los sistemas de trasporte utilizados por la comunidad académica de las Unidades Tecnológicas de Santander, UTS, en Bucaramanga, Santander, Colombia. Encuentro Internacional de Educación en Ingeniería, Bogotá, Colombia. https:// bit.ly/34GeL3g

Amaya, C.A., Hernández, C., Pedroza, N., y Cáceres, R.S. (2017). Estrategias de adaptación y mitigación al cambio climático de las Unidades Tecnológicas de Santander. Revista CINTEX, 22(2), 89-109. https://doi.org/10.33131/24222208.301

Área Metropolitana de Bucaramanga. (2011). Plan Maestro de Movilidad, Área Metropolitana de Bucaramanga 2011-2030. https://bit.ly/37MNayb

Área Metropolitana de Bucaramanga. (2019). Calidad del aire. https://bit.ly/3rtRv2i

Área Metropolitana del Valle de Aburra. (2019). Plan estratégico para la reducción de las emisiones de gases de efecto invernadero. https://bit.ly/2KhXeXQ 
Ayuntamiento de Murcia. (2012). Murcia, Ciudad Sostenible. Memoria a la candidatura al VII Premio Ciudad Sostenible. https://bit.ly/35y2dvE

Barton, J.R. (2009). Adaptación al cambio climático en la planificación de ciudades-regiones. Revista de geografía Norte Grande, (43), 5-30. http://dx.doi.org/10.4067/S0718$\underline{34022009000200001}$

Bastein, A., Verstraeten-Jochemsen, J., Rietveld, E., Hauck, M., Frijters, E., Klijn, O., \& Driessen, B. (2016). Circular Amsterdam. A vision and action agenda for the city and metropolitan area. Circle Economy- Gemeente Amsterdam. https://bit.ly/3IK9KwU

Bravo, E. (2018, 19 de mayo). Brasilia, la smart city del pasado. Tomorrow City. https://bit.ly/3f4tGYQ

Cariñanos, P., Calaza, P., Hiemstra, J., Pearlmutter, D., \& Vilhar, U. (2018). El papel de los bosques urbanos y periurbanos para reducir riesgos y gestionar desastres. Unasylva: Revista Internacional de Silvicultura e Industrias Forestales, 69(250), 53-59. https://bit.ly/3pnOX3L

Comisión Europea. (2010, 9 de marzo). Copenhague planifica su adaptación al cambio climático. https://bit.ly/32U1cMR

Corporación Autónoma para la Defensa de la Meseta de Bucaramanga. (2014). Plan de Gestión Ambiental Regional 2015-2030. https://bit.ly/2JzIPWL

Corporación Autónoma Regional del Tolima. (2018). Plan Integral de Gestión del Cambio Climático Territorial del Tolima. "Ruta Dulima. El Tolima enfrenta el cambio en el clima». https://bit. ly/2H9NmOX

de Rauglaudre, T. (2015, 15 de septiembre). Suecia: el líder mundial del medio ambiente (Trad. T. Bombassei. Le Journal International. https://bit.ly/35DhLOw

Delgado, G.C., de Luca, A., y Vázquez, V. (2015). Adaptación y mitigación urbana del cambio climático en México. Centro de Investigaciones Interdisciplinarias en Ciencias y Humanidades, Universidad Nacional Autónoma de México. https://bit.ly/36KBL1h

Departamento Nacional de Estadística. (2018). Censo poblacional y de vivienda de Colombia 2018. https://bit.ly/2IDLazM

Departamento Nacional de Planeación. (2012). Plan nacional de adaptación al cambio climático, Colombia. https://bit.ly/35FDXYE

Ecointeligencia. (2012). Ciudades Ecointeligentes. https://bit.ly/3/BCI25

Gobierno de la Ciudad de Buenos Aires. (2017). Informe de provincias. Adaptación de los Objetivos de Desarrollo Sostenible en la ciudad de Buenos Aires. https://bit.Iy/2K4F0c3

Grupo Intergubernamental de Expertos sobre el Cambio Climático. (2015). Cambio climático 2014: Informe de sintesis. Contribución de los Grupos de trabajo I, II y III al Quinto Informe de Evaluación del Grupo Intergubernamental de Expertos sobre el Cambio Climático. https:// bit.ly/3IGTWLA 
Hernández, C., Amaya, C.A., y Rodríguez, C.A. (2019, 24-29 de julio). Determinación del potencial de servicios ecosistémicos de la Comuna 7 en la ciudad de Bucaramanga Santander. Memory of 17th LACCEI International Multi-Conference for Engineering, Education, and Technology, Florida, Estados Unidos. http://dx.doi.org/10.18687/LACCEI2019.1.1.399

Hernández, R., Fernández, C., y Baptista, P. (2014). Metodología de la Investigación. (6ta ed.). Mac Graw Hill. https://bit.ly/2Kl8XFb

Instituto de Hidrología, Meteorología y Estudios Ambientales. (2016). Inventario nacional y departamental de gases efecto invernadero. Programa de las Naciones Unidas para el Desarrollo. https://bit.ly/3azZDIx

Margulis, S. (2016). Vulnerabilidad y adaptación de las ciudades de América Latina al cambio climático. Comisión Económica para América Latina y el Caribe. https://bit.ly/2IHImCJ

Ministerio de Ambiente y Desarrollo Sostenible. (2016). Plan Integral de Gestión del Cambio Climático Territorial del Santander 2030. https://bit.ly/2IKYySq

Morán, N. (2010). La Red Ecológica de Bolonia (Italia). Un sistema territorial de nodos y corredores verdes que recupera las estructuras rurales y el paisaje agrario tradicional. En M. Vázquez y C. Verdaguer (Eds.), El espacio agrícola entre el campo y la ciudad (págs. 51-61). Grupo de Investigación en Arquitectura, Urbanismo y Sostenibilidad. https://bit.ly/3pArvkU

Muévete en Verde. (2018). Valencia: una ciudad comprometida con la movilidad sostenible. Recuperado en marzo 2019 de https://bit.ly/37K2vki

Nahuelhual, L., Laterra, P., y Barrena, J. (2016). Indicadores de servicios ecosistémicos: una revisión y análisis de su calidad. Ministerio del Medio Ambiente de Chile. https://bit.ly/35z5NWn

Oltra, Ch., y Marín, R. (2013). Los retos en la adaptación al cambio climático en entornos urbanos. Revista de Sociología, 98(2), 311-330. http://dx.doi.org/10.5565/rev/papers/v98n2.498

Organización de las Naciones Unidas para la Alimentación y la Agricultura. (2016). Agricultura urbana y periurbana en América Latina y el Caribe. https://bit.ly/32TU5nD

Organización de las Naciones Unidas. (2019). Conferencia de las Naciones Unidas sobre el Cambio Climático (COP 25). https://bit.ly/3f8roYW

Organización de Naciones Unidas. (1992). Convención Marco de Naciones Unidas sobre Cambio Climático. https://bit.ly/2KUzfOW

Organización de Naciones Unidas. (2015). Transformar nuestro mundo: la Agenda 2030 para el Desarrollo Sostenible. https://bit.ly/2UE9uDX

Organización de Naciones Unidas. (2017). Nueva Agenda Urbana, Hábitat III. https://bit.ly/33Hj1PE 
Pérez, C. (2016). Análisis de vulnerabilidad y planes de adaptación urbana para la reducción de los efectos del cambio climático en Medellín [Tesis de maestría, Universidad Nacional de Colombia]. Repositorio Institucional UNC. https://bit.Iy/2LVOKYO

Robrecht, H. (2019, 10 de diciembre). El cambio climático y las ciudades. Agencia Europea del Medio Ambiente. https://bit.ly/2ILiRPE

Sánchez, R. (Ed.). (2013). Respuestas urbanas al cambio climático en América Latina. Comisión Económica para América Latina y el Caribe-Instituto Interamericano para la Investigación del Cambio Global. https://bit.ly/2UzFmcU

Sengupta, S. (2019, 26 de marzo). Copenhague, un ejemplo en el combate al cambio climático. The New York Times. https://nyti.ms/3IDrmdL

Universidad Industrial de Santander. (2018). Línea base para los Objetivos de Desarrollo Sostenible Santander. Programa de Naciones Unidas para el Medio Ambiente. https://bit.ly/3nvXhOy

Veeduría Distrital. (2019). Concretando la agenda de adaptación al cambio climático en Bogotá. Alcaldía de Bogotá. https://bit.ly/2IID6NR 